\title{
Compassionate Use of Opaganib for Patients with Severe COVID-19
}

\author{
Ramzi Kurd ${ }^{1, \#, ~ E l i ~ B e n-C h e t r i t ~}{ }^{2,3, \#}$, Hani Karameh ${ }^{1}$ and Maskit Bar-Meir ${ }^{2,3, *}$ \\ ${ }^{1}$ Internal medicine, Shaare-Zedek Medical Center, Jerusalem, Israel \\ ${ }^{2}$ Infectious diseases, Shaare-Zedek Medical Center, Jerusalem, Israel \\ ${ }^{3}$ The Faculty of Medicine, Hebrew University, Jerusalem, Israel \\ "These authors contributed equally to this article
}

*Corresponding authors: Maskit Bar-Meir, MD, MSc, Infectious diseases, Shaare-Zedek Medical Center, Jerusalem, Israel 91031, Tel: +972-508685507; Fax: +972-2-6555197; E-mail: mbarmeir@gmail.com

Received: 21 Sep, 2020 | Accepted: 04 Nov, 2020 | Published: 11 Nov, 2020

Citation: Kurd R, Ben-Chetrit E, Karameh H, Bar-Meir M (2020) Compassionate Use of Opaganib for Patients with Severe COVID-19. J Emerg Dis Virol 5(3): dx.doi.org/10.16966/2473-1846.159

Copyright: (c) 2020 Kurd R, et al. This is an open-access article distributed under the terms of the Creative Commons Attribution License, which permits unrestricted use, distribution, and reproduction in any medium, provided the original author and source are credited.

\section{Abstract}

Opaganib is a Sphingosine-kinase (SK)-2 inhibitor with anti-inflammatory and anti-viral properties. Five severe COVID-19 patients were treated with opaganib on compassionate-use basis. Treatment was well tolerated and associated with faster lymphocyte count increase and improved oxygenation. Opaganib efficacy for COVID-19 should be examined in a clinical trial setting.

Keywords: COVID-19; Opaganib; Compassionate-use

\section{Introduction}

Opaganib (RedHill Biopharma Ltd.) is a selective SK-2 inhibitor with anti-inflammatory and anti-viral properties. Sphingomyelin is the precursor of ceramide and the proinflammatory lipid sphingosine 1-phosphate (S1P). Increasing intracellular S1P levels and depletion of ceramide promote cell proliferation and inhibit apoptosis. Platelets, monocytes, and mast cells secrete S1P upon activation, thus promoting inflammatory cascades $[1,2]$. Moreover, SK activation is essential for the signaling responses to Tumor Necrosis Factor alpha [3]. Opaganib (ABC294640) is an orally available SK-2 inhibitor noted to have anti-inflammatory activity in rodent models of arthritis and inflammatory bowel disease [4-6] as well as antiviral effects. Impairment of SK-2 function significantly inhibited Chikungunya virus infection [7], decreased viral titers of Influenza in an in-vitro model [8] and demonstrated inhibitory effect in Ebola cell-based inhibition assay [9].

Three clinical trials have been completed with opaganib, a phase 1 study in advanced solid tumor patients [10], a food effect study in healthy volunteers and a phase $1 b / 2$ in patients with advanced multiple myeloma (unpublished). Two more studies are in progress, a phase 2 study in advanced prostate cancer (clinicaltrials.gov ID: NCT04207255) and phase 2 study in cholangiocarcinoma (clinicaltrials.gov ID: NCT03377179).

Here we report our experience with this treatment in COVID-19 patients and compare patients' outcomes to those of untreated patients with similar disease severity.

\section{The Study}

Shaare-Zedek Medical Center (SZMC) is a tertiary academic hospital in Jerusalem, IL. On February $27^{\text {th }}$ the first case of COVID-19 was diagnosed in Israel, with a dramatic increase in the number of cases following a Jewish holiday in the middle of March. Many cases were diagnosed in the orthodox Jewish communities of Jerusalem, which the hospital serves.

RedHill Biopharma, Ltd. offered opaganib under compassionate use for the treatment of COVID-19 patients. Eligible patients were those hospitalized with severe COVID-19, requiring oxygen support via high-flow nasal cannula (HFNC). Intubated or debilitated patients, pregnant or nursing women, patients on oral anti-coagulants or evidence of ischemia on ECG were excluded. In addition to signing the informed consent, patients were required to have acceptable liver and renal function. Oral opaganib was delivered at a dose of $500 \mathrm{mg}$ q12 hours for up to 14 days, or until patient discharge. Additional therapy was allowed as deemed necessary by the clinicians. Data were collected through May 5, 2020.

\section{Assessment and analysis}

Clinical data were collected daily until patient discharge. For comparison, we used a control group with same-sex, same-severity (male patients requiring HFNC oxygen support), aged 35 to 80 years, who met the inclusion and exclusion criteria. These patients may have been eligible for treatment, but were not approached due to time and staff constraints. 


\section{Ethical considerations}

Individual approval was granted for each patient treated with opaganib by the SZMC institutional review board (IRB) and the Israeli Ministry of Health. Written informed consent was obtained from each participant. For the control group, IRB approval was granted to collect de-identified data. RedHill Biopharma Ltd. provided the medication, but was not involved in the conduct of treatment or follow-up, nor in the data collection and analyses.

Univariate comparisons between the groups were performed with chi-square test for categorical variables and t-test or Mann-Whitney U-test for continuous variables, as appropriate. Time variables were compared with Cox proportional hazard regression, adjusted for age and background illnesses. C-reactive protein (CRP) and lymphocyte counts were recorded at day 1 (treatment or admission) and at interval of 2-4 days, and compared with repeated measures general linear model with Bonferroni correction for multiple comparisons. All analyses were conducted with SPSS software, version 25.0 (IBM).

Seven patients received at least one dose of opaganib since April 2, 2020. All patients received Hydroxychloroquine (HCQ), however one stopped HCQ prior to opaganib treatment due to borderline Q-T interval in ECG. Three patients received azithromycin as well. One patient, who received both HCQ and azithromycin, developed diarrhea after two doses of opaganib, and the treating physicians decided to stop all his medications. A second patient was discharged after receiving two doses of opaganib. Therefore, five patients were included in this analysis. Overall, 3 received the full 14-day course of opaganib, and 2 patients received 11 and 7 days respectively, before being discharged. All patients who received opaganib were male (as approximately two-thirds of severe patients in SZMC).

\section{Safety}

Except for diarrhea in one patient, no other adverse events related to the medication were observed. One patient reported dysuria on day 12 of treatment and another patient had an episode of atrial fibrillation that resolved spontaneously.

\section{Baseline characteristics}

Median age, rates of diabetes, hypertension and obesity, as well as median duration of symptoms prior to admission were similar between treatment and control groups (Table 1). Cases had somewhat higher baseline lymphocyte counts and lower D-dimer levels compared with controls. All patients received HCQ, and the majority also received azithromycin. However, methylprednisolone was given to a third of controls and to none of cases.

\section{Clinical and laboratory outcomes}

Lymphocyte counts increased significantly faster in the treatment group ( $\mathrm{p}=0.001$, Figure 1 ). Of note, a third of the control patients received systemic steroids. In order to exclude bias related to the lymphocytopenic effect of steroids, the general linear model was performed again excluding these 6 patients, yielding similar results $(\mathrm{p}=0.013)$.

All other outcomes trended in favor of the opaganib group, however did not reach statistical significance: CRP decreased faster among cases $(\mathrm{p}=0.08$, Figure 2$)$, median time to weaning from HFNC (time to NC) was 10 days (IQR: 9-15.5) compared with 15 days (IQR: 8-19) in cases vs. controls [Hazard ratio $(\mathrm{HR})=0.3,95 \%$ CI: 0.07-1.7, $\mathrm{p}=0.2$ ] and time to breathing ambient air was 13 days in the opaganib group (IQR: 10.5-19) vs. 14.5 days in the controls (IQR: 10-28); $(\mathrm{HR}=0.4$, 95\% CI: 0.15-1.5).
Table 1: Baseline characteristics of the patients*.

\begin{tabular}{|c|c|c|c|}
\hline Characteristic & $\begin{array}{l}\text { Opaganib } \\
(\mathrm{N}=5)\end{array}$ & $\begin{array}{l}\text { Control } \\
(\mathrm{N}=18)\end{array}$ & $p$ \\
\hline Median age $\left(\mathrm{IQR}^{*}\right)$, Years & $58(50-73.2)$ & $58.5(54.5-66)$ & 0.9 \\
\hline $\begin{array}{l}\text { Median duration of symptoms } \\
\text { before admission (IQR), days }\end{array}$ & $7(6-8)$ & $6(2.5-8)$ & 0.5 \\
\hline \multicolumn{4}{|l|}{ Coexisting conditions-no.(\%) } \\
\hline Hypertension & $2(40)$ & $6(33)$ & 1 \\
\hline Diabetes Mellitus & $2(40)$ & $6(33)$ & \\
\hline Obesity & $2(40)$ & $8(44)$ & \\
\hline \multicolumn{4}{|l|}{$\begin{array}{l}\text { Baseline laboratory values, } \\
\text { Median (IQR) }\end{array}$} \\
\hline $\mathrm{CRP}, \mathrm{mg} / \mathrm{dL}$ & $\begin{array}{c}15.8(12.6- \\
21.7)\end{array}$ & $18.6(9-26.7)$ & 0.6 \\
\hline Lymphocytes, $\mathrm{mm}^{3}$ & $\begin{array}{c}1100(995- \\
1300)\end{array}$ & $850(635-970)$ & 0.09 \\
\hline Fibrinogen, mg/dL & $\begin{array}{c}858(844- \\
2746)\end{array}$ & $741(676-828)$ & 0.04 \\
\hline D-dimer, ng/mL & $\begin{array}{c}1208(531- \\
2633)\end{array}$ & $\begin{array}{c}1578(1073- \\
3734)\end{array}$ & 0.07 \\
\hline Ferritin, ng/mL & $\begin{array}{c}1483(999- \\
5180)\end{array}$ & 758 (530-1109) & 0.3 \\
\hline \multicolumn{4}{|l|}{$\begin{array}{l}\text { Concomitant } \quad \text { medications } \\
\text { given for COVID-19, } \mathrm{N}(\%)\end{array}$} \\
\hline Hydroxychloroquine & $5(100)$ & $18(100)$ & 1 \\
\hline Azithromycin & $3(60)$ & $14(77)$ & 0.5 \\
\hline Methylprednisolone & 0 & $6(33)$ & 0.2 \\
\hline $\begin{array}{l}\text { Cycle threshold (Ct) value } \\
\text { In RT-PCR of nasal swabs** }\end{array}$ & $24.8 \pm 2.6$ & $25.9 \pm 5.5$ & 0.8 \\
\hline
\end{tabular}

*All patients are male; IQR-Interquartile range

**Values of cycle threshold are an average of $\mathrm{E}, \mathrm{N}$ and RdRp genes tested

\section{Complications}

All patients in the treatment group were discharged by the time of data collection; none have required mechanical ventilation or suffered complications. Among controls, 5(27\%) patients were not discharged by the time of data collection, $6(33 \%)$ required mechanical ventilation ( $\mathrm{p}$ value compared with opaganib group=0.13), 2 (11\%) required $\mathrm{ECMO}$, and 1 required tracheostomy. None of the patients in both groups had died by the time of data collection.

\section{Conclusions}

At the time of this study, no effective treatment was available for COVID-19 infection. Only at the end of this data collection, a preliminary report was published which showed some improvement in clinical outcomes of severe COVID-19 patients treated with remdesivir [11]. Neither remdesivir nor other potential treatments such as convalescent plasma, were available for our patients. The anti-inflammatory and anti-viral properties of opaganib along with a good safety profile motivated us to offer it under compassionate use to severe COVID-19 patients.

Patients treated with opaganib had significantly faster increase in lymphocyte count. Low lymphocyte counts were associated with a more severe COVID-19 disease [12] and with faster disease progression $[13,14]$; therefore this faster increase may indicate better prognosis for the treatment group. Interestingly, the faster lymphocyte 


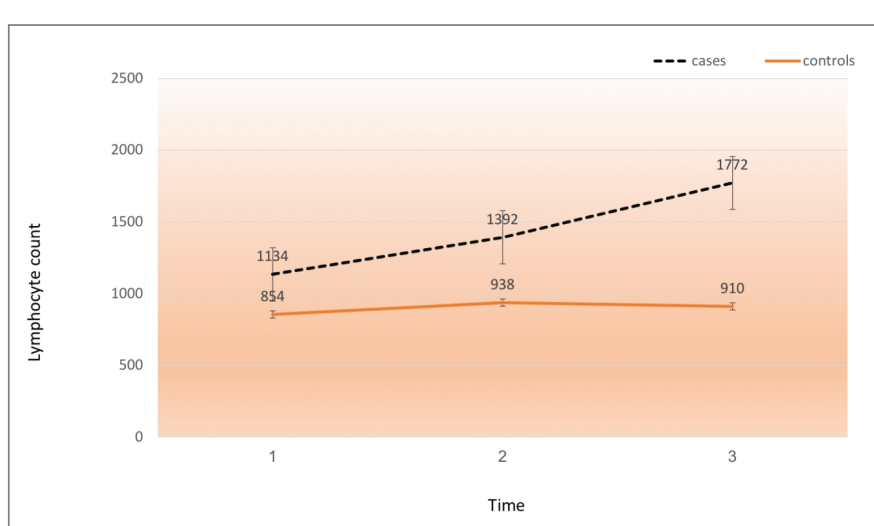

Figure 1: Lymphocytes (counts $/ \mathrm{mm}^{3}$ ) increase in cases (dashed line) vs. controls (solid line) over three time points (day of admission or day of treatment start and every 2-4 days), $p=0.001$.

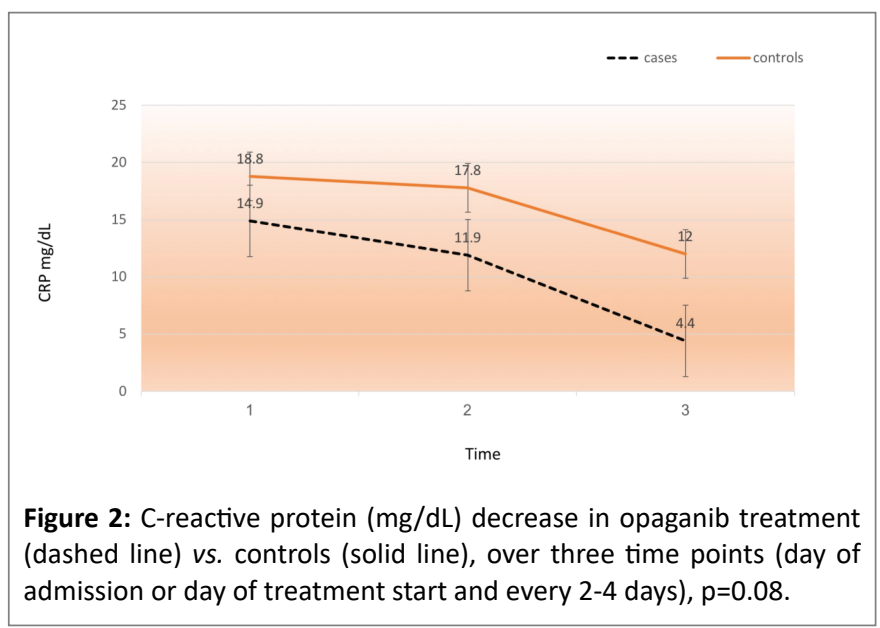

recovery among opaganib treated patients persisted even after excluding control patients who received systemic steroids. Although other clinical outcomes did not differ significantly between groups, the trends of time to improved oxygenation, rate of CRP decrease, as well as rates of mechanical ventilation and complications were all in favor of the treatment group.

Opaganib treatment was well tolerated. Only in one patient was treatment stopped, due to diarrhea, while being treated with HCQ and azithromycin in addition to opaganib.

The results of the present report are preliminary and are limited by the small number of patients. However, these data justify examining the effect of opaganib in the setting of a clinical trial, with larger numbers of patients and, potentially, with moderate disease severity.

\section{Conflict of Interest Statement}

All authors declare no conflict of interest.

\section{References}

1. Yatomi Y, Ruan F, Hakomori S, Igarashi Y (1995) Sphingosine-1phosphate: a platelet-activating sphingolipid released from agoniststimulated human platelets. Blood 86: 193-202.

2. Prieschl EE, Csonga R, Novotny V, Kikuchi GE, Baumruker T (1999) The balance between sphingosine and sphingosine-1-phosphate is decisive for mast cell activation after Fc epsilon receptor I triggering. J Exp Med 190: 1-8.

3. Xia P, Gamble JR, Rye KA, Wang L, Hii CST, et al. (1998) Tumor necrosis factor- $\alpha$ induces adhesion molecule expression through the sphingosine kinase pathway. Proc Natl Acad Sci U S A 95: 1419614201.

4. Maines LW, Fitzpatrick LR, French KJ, Zhuang Y, Xia Z, et al. (2008) Suppression of ulcerative colitis in mice by orally available inhibitors of sphingosine kinase. Dig Dis Sci 53: 997-1012.

5. Maines LW, Fitzpatrick LR, Green CL, Zhuang Y, Smith CD (2010) Efficacy of a novel sphingosine kinase inhibitor in experimental Crohn's disease. Inflammopharmacology 18: 73-85.

6. Fitzpatrick LR, Green C, Frauenhoffer EE, French KJ, Zhuang Y, et al. (2011) Attenuation of arthritis in rodents by a novel orally-available inhibitor of sphingosine kinase. Inflammopharmacology 19: 75-87.

7. Reid SP, Tritsch SR, Kota K, Chiang CY, Dong L, et al. (2015) Sphingosine kinase 2 is a chikungunya virus host factor co-localized with the viral replication complex. Emerg Microbes Infect 4: e61.

8. Xia C, Seo YJ, Studstill CJ, Vijayan M, Wolf JJ, et al. (2018) Transient inhibition of sphingosine kinases confers protection to influenza $A$ virus infected mice. Antiviral Res 158: 171-177.

9. Reza F, Dror BA, Guy G (2020) Therapy for inhibition of singlestranded RNA virus replication. US-2016113954-A1, Redhill Biopharma Ltd (IL).

10. Britten CD, Garrett-Mayer E, Chin SH, Shirai K, Ogretmen B, et al. (2017) A Phase I Study of ABC294640, a First-in-Class Sphingosine Kinase-2 Inhibitor, in Patients with Advanced Solid Tumors. Clin Cancer Res 23: 4642-4650.

11. Grein J, Ohmagari N, Shin D, Diaz G, Asperges E, et al. (2020) Compassionate Use of Remdesivir for Patients with Severe COVID-19. N Engl J Med 382: 2327-2336.

12. Wynants L, Van Calster B, Collins GS, Riley RD, Heinze G, et al. (2020) Prediction models for diagnosis and prognosis of COVID-19 infection: systematic review and critical appraisal. BMJ 369: m1328.

13. Zhang J, Yu M, Tong S, Liu LY, Tang LV (2020) Predictive factors for disease progression in hospitalized patients with coronavirus disease 2019 in Wuhan, China. J Clin Virol 127: 104392.

14. Yuan C, Gaskins AJ, Blaine Al, Zhang C, Gillman MW, et al. (2016) Association between Cesarean Birth and Risk of Obesity in Offspring in Childhood, Adolescence, and Early Adulthood. JAMA Pediatr 170: e162385. 